\title{
Efficacy of trastuzumab in Japanese patients with HER2-positive advanced gastric or gastroesophageal junction cancer: a subgroup analysis of the Trastuzumab for Gastric Cancer (ToGA) study
}

\author{
Akira Sawaki • Yasuo Ohashi • Yasushi Omuro • Taroh Satoh • Yasuo Hamamoto • Narikazu Boku • \\ Yoshinori Miyata $\cdot$ Hiroya Takiuchi $\cdot$ Kensei Yamaguchi $\cdot$ Yasutsuna Sasaki $\cdot$ Tomohiro Nishina \\ Atsushi Satoh $\cdot$ Eishi Baba $\cdot$ Takao Tamura $\cdot$ Takashi Abe $\cdot$ Kiyohiko Hatake $\cdot$ Atsushi Ohtsu
}

Received: 6 August 2011/Accepted: 31 October 2011/Published online: 17 December 2011

(c) The Author(s) 2011. This article is published with open access at Springerlink.com

\begin{abstract}
Background The Trastuzumab for Gastric Cancer (ToGA) study is the first international trial to include Japanese patients with human epidermal growth factor 2 (HER2) positive advanced/metastatic gastric or gastroesophageal junction cancer. ToGA showed that trastuzumab plus chemotherapy (capecitabine/cisplatin or 5-fluorouracil/cisplatin) improved overall survival in the overall population (hazard ratio 0.74 ).
\end{abstract}

Presented in part at the American Society of Clinical Oncology Gastrointestinal Cancer Symposium, San Francisco, 20-22 January 2011.

\section{A. Sawaki}

Department of Gastroenterology, Aichi Cancer Center Hospital,

Aichi, Japan

\author{
A. Sawaki $(\square)$ \\ Division of Oncology, Nagoya Daini Red Cross Hospital, \\ 2-9 Myoukenchou Shouwa-ku, Nagoya 466-8650, Japan \\ e-mail: sawaki@jk2.so-net.ne.jp \\ Y. Ohashi \\ Department of Biostatistics, Public Health Research Foundation, \\ Tokyo, Japan \\ Y. Omuro \\ Department of Chemotherapy, Tokyo Metropolitan Cancer and \\ Infectious Diseases Center Komagome Hospital, Tokyo, Japan \\ T. Satoh \\ Department of Medical Oncology, Kinki University Faculty \\ of Medicine, Osaka, Japan \\ Y. Hamamoto \\ Department of Medical Oncology, Tochigi Cancer Center, \\ Tochigi, Japan \\ N. Boku \\ Division of Gastrointestinal Oncology, Shizuoka Cancer Center, \\ Shizuoka, Japan
}

Regional differences in outcome in favor of Japanese populations were observed in other studies; therefore, subgroup analyses of ToGA may contribute to the evaluation of the potential benefits of this regimen in Japanese patients.

Methods We performed subgroup analyses on 101 Japanese patients enrolled into ToGA (trastuzumab plus chemotherapy, $n=51$; chemotherapy, $n=50$ ).

Results Median overall survival in the Japanese subgroup was 15.9 months (95\% confidence interval 12-25) for trastuzumab plus chemotherapy and 17.7 months (95\% confidence interval 12-24) for chemotherapy (hazard ratio $1.00 ; 95 \%$ confidence interval $0.59-1.69$ ). After adjusting

\section{Y. Miyata}

Department of Gastroenterology, Saku Central Hospital,

Nagano, Japan

H. Takiuchi

Second Department of Internal Medicine, Osaka Medical

College, Osaka, Japan

K. Yamaguchi

Division of Gastroenterology, Saitama Cancer Center,

Saitama, Japan

Y. Sasaki

Department of Medical Oncology, Saitama International

Medical Center-Comprehensive Cancer Center,

Saitama Medical University, Saitama, Japan

T. Nishina

Department of Gastroenterology, National Hospital Organization

Shikoku Cancer Center, Ehime, Japan

A. Satoh

Department of Internal Medicine, Toyosu Hospital,

Showa University School of Medicine, Tokyo, Japan

E. Baba

Department of Hematology and Oncology,

Kyushu University Hospital, Fukuoka, Japan 
for prespecified covariates, the estimated hazard ratio for overall survival was $0.68 \quad(95 \%$ confidence interval 0.36-1.27). Further post hoc and exploratory examinations supported the robustness of the adjusted hazard ratios.

Conclusions After adjusting for imbalanced patient backgrounds between arms, overall survival of Japanese patients with human epidermal growth factor 2 positive advanced/ metastatic gastric or gastroesophageal junction cancer who received trastuzumab plus chemotherapy was improved compared with patients who received chemotherapy alone.

Keywords Trastuzumab - Drug therapy - Stomach neoplasms · Randomized controlled trial

\section{Background}

Approximately 110,000 people in Japan develop gastric cancer each year [1], with 65,000 estimated deaths (which is second only to lung cancer among cancer-related deaths [1]). For advanced disease, the oral fluoropyrimidine $S-1$, in combination with cisplatin, has become the standard treatment for gastric cancer in Japan, based on the results of the SPIRITS trial [2]. However, the prognosis still remains poor, and therefore new therapies such as molecular-targeted drugs are needed. Trastuzumab is a recombinant monoclonal antibody that targets human epidermal growth factor receptor 2 (HER2). Trastuzumab derives its anticancer effects from inducing antibody-dependent cytotoxicity, inhibiting HER2-mediated signaling, and preventing cleavage of the extracellular domain of HER2 [3].

Trastuzumab has been approved for use in HER2-positive metastatic breast cancer and as a postoperative adjuvant therapy for HER2-positive breast cancer, and is now the standard of care worldwide for these indications, including in Japan. The Trastuzumab for Gastric Cancer (ToGA) study was the first international randomized controlled phase III trial to include Japanese patients with HER2-positive advanced/metastatic gastric or gastroesophageal junction

\section{T. Tamura}

Division of Diabetes, Digestive, and Kidney Diseases, Department of Clinical Molecular Medicine, Kobe University Graduate School of Medicine, Hyogo, Japan

T. Abe

Internal Medicine, Yamagata Prefectural Central Hospital,

Yamagata, Japan

\section{K. Hatake}

Medical Oncology/Hematology, JFCR Cancer Institute Ariake Hospital, Tokyo, Japan

A. Ohtsu

Research Center for Innovative Oncology, National Cancer Center Hospital East, Chiba, Japan
(GEJ) cancer. The percentage of patients with HER2-positive gastric cancer, as assessed by immunohistochemistry (IHC; $3+$ on a scale of 0 to $3+$ ) or fluorescence in situ hybridization (FISH; HER2:CEP17 ratio $\geq 2.0$ ) was $22.1 \%$ in the overall ToGA population. The proportion of patients with HER2positive disease was similar for Europe (23.6\%), Asia (23.5\%), and Japan (27.6\%) [4], and similar to that seen in patients with breast cancer in other trial populations (25-30\%) [5]. ToGA showed that patients who received combination treatment with trastuzumab and chemotherapy [capecitabine plus cisplatin (XP) or fluorouracil plus cisplatin (FP)] had significantly improved survival compared with those who received chemotherapy alone: the median overall survival (OS) in the intent-to-treat (ITT) population was 13.8 months in the trastuzumab plus chemotherapy arm and 11.1 months in the chemotherapy-only arm [hazard ratio (HR) $0.74,95 \%$ confidence interval (CI) $0.60-0.91$; $P=0.0046]$ [6].

There were substantial differences in OS reported from recent phase III trials of chemotherapy for gastric cancer, and these are especially evident between Japan and other countries. Recent trials in Japan have demonstrated that combination therapy resulted in longer survival than was seen in studies outside of Japan, with a median survival exceeding 1 year [7, 8], as compared with around 10 months in Western trials $[9,10]$. There are considered to be two reasons for the longer survival observed in Japanese trials. Firstly, up to $70 \%$ of Japanese patients receive subsequent chemotherapy following failure of first-line therapy [11-13]. Secondary, there may be differences in the eligibility criteria and baseline patient characteristics between the Japanese and non-Japanese trials; the studies in Japan included patients with and without measurable metastatic disease, whereas non-Japanese trials usually included patients with measurable metastatic disease only [11]. Since the primary endpoint of the ToGA study was $\mathrm{OS}$, there is a possibility that the impact of trastuzumab on survival might be reduced in Japanese patients due to inherently longer survival in this population. To evaluate the efficacy of trastuzumab in combination with chemotherapy specifically in the Japanese population of ToGA, we conducted preplanned and post hoc subgroup analyses.

\section{Patients and methods}

The details of the ToGA trial design and methods have been reported elsewhere [6].

Japanese patient subgroup

To evaluate the efficacy and safety of the combination treatment (trastuzumab plus XP) in the Japanese population 
of the ToGA study, we performed subgroup analyses using data from patients who were enrolled from institutions in Japan.

Preplanned sample size for Japanese patients

In the ToGA study, the HR for OS was expected to be 0.77 , the expected number of events was 460 , and the target sample size was set at 584 patients [6]. Before starting the ToGA study, we set the sample size of Japanese patients to allow us to evaluate similarities between the overall ToGA results and our subgroup analysis in an exploratory manner. Assuming a $70 \%$ probability that the HR for OS in the Japanese subgroup would be less than 0.88 (the midpoint between 0.77 and 1.00), the expected number of events was 70 . To reach this expected number of events within the study period, the minimum sample size was determined to be 89 patients to allow us to conduct four analyses: preplanned (unadjusted and adjusted), post hoc, and exploratory analyses of the HR.

Unadjusted analyses

We calculated the unadjusted OS and progression-free survival (PFS) of the Japanese sub-group using the same methods as those used for the overall ToGA study [6]. Objective response rate of the Japanese sub-group was analysed with a $\chi^{2}$ test in patients with measurable disease $(n=45$ in the trastuzumab plus XP arm and 41 in the XP arm).

\section{Preplanned analyses}

Prior to carrying out the Japanese subgroup analysis, we predicted an imbalance in the baseline patient characteristics. Therefore, we planned to calculate an adjusted $\mathrm{HR}$ and $95 \% \mathrm{CI}$ in the Japanese subgroup using a multivariate Cox regression analysis with 15 factors: extent of disease, primary tumor site, measurability of disease, Eastern Cooperative Oncology Group Performance Status (ECOG PS), chemotherapy regimen (stratification factors), sex, age, number of lesions, number of metastatic sites, type of gastric cancer, visceral metastasis, prior gastrectomy, prior chemotherapy, HER2 status, and region of origin (other prespecified covariates). All factors were prespecified in the ToGA study protocol. Each covariate was also evaluated using a univariate Cox regression analysis.

Post hoc analyses

During the preplanned multivariate Cox regression analysis, we excluded patients for whom HER2 status was reported as IHC 3+/FISH unknown (no result). In addition, estimates of effects were extremely unstable for covariates that contained a category which included only one patient. Therefore, to target all of the enrolled patients and ensure the stability of the model, a post hoc analysis was conducted using a multivariate Cox analysis. Among covariates, HER2 status was divided into two categories: high expression (IHC $2+$ and FISH-positive or IHC 3+) and low expression (IHC0 and FISH-positive or IHC $1+$ and FISH-positive). Covariates that contained a category with only one patient (extent of disease and previous chemotherapy) were excluded from the model to ensure its stability.

Exploratory analyses to evaluate deviation of patient prognosis

To identify factors that affect prognosis specifically in the Japanese subgroup, and to confirm the robustness of our preplanned and post hoc analyses, an exploratory multivariate Cox regression analysis on the HR for OS with various combinations of covariates was carried out. We created a series of models that included the treatment group as a base covariate with 3-6 other covariates, and selected the top four models ranked by value following a chi-square test. The procedure was repeated for the models with three, four, five, and six covariates, and a total of 16 models were selected. From the well-fitting model that was obtained, we compared the HR for OS with the results of preplanned and post hoc analyses. To ensure that HER2 status was not a confounding variable, we carried out a multivariate Cox regression analysis with HER2 expression (high or low) as the stratification factor, and determined the HR for OS in which selected covariates were included in the model.

Furthermore, scoring of the prognosis of each patient in both study arms using the Cox regression model and estimation of the risk for each patient were carried out with the selected covariates. The risk was shown by the estimated value of logarithm HR for each patient. To eliminate the influence of treatment on the mortality risk, we set the treatment group as the stratification factor and produced a histogram plot according to the distribution of patient risk to evaluate potential bias between the treatment arms.

\section{Safety}

Adverse events and serious adverse events were assessed according to the National Cancer Institute Common Terminology Criteria for Adverse Events (NCI-CTCAE) version 3.0 and the International Conference on Harmonization guidelines, respectively.

\section{Results}

Patients

Between September 2005 and December 2008, 594 patients were enrolled in the primary ToGA study at 122 
Table 1 HER2 testing results in the Japanese population of ToGA

\begin{tabular}{lrllll}
\hline FISH result & \multicolumn{5}{l}{ IHC score } \\
\cline { 2 - 6 } & IHC 0 & IHC 1+ & IHC 2+ & IHC 3+ & Total \\
\hline FISH-positive, $n$ & 14 & 19 & 36 & 37 & 106 \\
FISH-negative, $n$ & 155 & 57 & 14 & 1 & 227 \\
NE, $n$ & 48 & 12 & 8 & 8 & 83 \\
Total, $n$ & 217 & 88 & 58 & 46 & 409 \\
\hline
\end{tabular}

FISH fluorescence in situ hybridization, HER2 human epidermal growth factor receptor 2, IHC immunohistochemistry, $N E$ not evaluable

centers in 24 countries, of whom 584 were included in the primary analysis. Four hundred twenty-one tumor samples were provided for HER2 testing from 16 centers in Japan. Twelve samples were not evaluated due to a lack of tumor tissue in the sample $(n=7)$, shipment failure $(n=4)$, or disease progression before shipment $(n=1)$. Of the 409 samples successfully screened, $115(28.1 \%)$ were scored as HER2-positive (IHC 3+ or FISH-positive; Table 1) and 102 patients were registered into the study. After excluding one patient who did not receive the study drug, 101 Japanese patients (trastuzumab plus chemotherapy, $n=51$; chemotherapy alone, $n=50$ ) were included in this subgroup analysis. All patients received capecitabine as the chemotherapy partner of cisplatin.

Table 2 shows the baseline characteristics of the Japanese patients included in this subgroup analysis $(n=101)$ and the non-Japanese patients $(n=483)$. There is similarity in the baseline characteristics of patients from other countries between the study arms. On the other hand, number of metastatic sites, histologic type, and prior gastrectomy were imbalanced by approximately $10 \%$ between the study arms in the Japanese subgroup, and were considered to be prognostic factors. Median follow-up times were 18.6 months [interquartile range (IQR) 11-25] in the trastuzumab plus XP arm and 17.1 months (IQR 1-49) in the XP arm. The median number of cycles of trastuzumab therapy was eight (range 1-24). Forty-one patients in the trastuzumab plus XP arm (80.4\%) and 41 patients in the XP arm $(82.0 \%)$ received second-line treatment (at least one chemotherapy treatment after disease progression despite the study treatments).

\section{Efficacy}

Unadjusted analyses

Twenty-eight patients (54.9\%) in the trastuzumab plus XP arm and 27 patients (54.0\%) in the XP arm had died by the data cutoff point. As shown in Table 3, unadjusted median OS was 15.9 months (95\% CI 12-25 months) in the trastuzumab plus XP arm and 17.7 months $(95 \%$ CI 12-24 months) in the XP arm (HR 1.00, 95\% CI 0.59-1.69). The number of PFS events (defined as disease progression or death) was $43(84.3 \%)$ in the trastuzumab plus XP arm and $40(80.0 \%)$ in the XP arm. Unadjusted median PFS was 6.2 months (95\% CI 5-7 months) in the trastuzumab plus XP arm and 5.6 months (95\% CI 5-7 months) in the XP arm (HR 0.92, 95\% CI 0.60-1.43). The objective response rate was $64.4 \% \quad(95 \%$ CI 48.8-78.1\%) in the trastuzumab plus XP arm and 58.5\% (95\% CI 42.1-73.7\%) in the XP arm.

\section{Preplanned analyses}

In the multivariate analysis, the HR for OS, adjusted by the 15 prespecified covariates above, was 0.68 (95\% CI $0.36-1.27, P=0.2251$, Table 4$)$. The adjusted HR for PFS was 0.66 (95\% CI $0.40-1.09 \%$ ), which was slightly improved compared with the results for the overall population. Among the covariates in the preplanned analysis, the univariate analysis showed that prior gastrectomy was the covariate most strongly associated with longer OS (HR $0.39,95 \%$ CI $0.16-0.91$ ). There were more patients with prior gastrectomy in the XP arm (26\%) than in the trastuzumab arm (16\%). After adjusting for gastrectomy only, the HR for OS between the treatment arms was $0.85(95 \%$ CI 0.49-1.45).

\section{Post hoc analyses}

For the post hoc exploratory multivariate Cox regression analysis, the adjusted HRs for OS and PFS were $0.82(95 \%$ CI 0.45-1.50) and 0.81 (95\% CI 0.50-1.30), respectively (Fig. 1).

Exploratory analyses to evaluate deviation of patient prognosis

We evaluated the HR for OS with different combinations of covariates in the model. In the well-fitting models with high chi-square values, the HRs using three, four, five, and six covariates ranged between 0.79 (95\% CI $0.49-1.38$ ) and 0.89 (95\% CI 0.52-1.54), 0.77 (95\% CI 0.44-1.33) and 0.88 (95\% CI $0.51-1.53), 0.68$ (95\% CI $0.39-1.20)$ and 0.80 (95\% CI $0.45-1.42$ ), and 0.68 (95\% CI $0.38-1.20$ ) and 0.76 (95\% CI 0.44-1.33), respectively. In choosing the well-fitting models, the covariates sex, HER2 status, type of gastric cancer, prior gastrectomy, prior chemotherapy, and number of lesions tended to be chosen. The sets of covariates were similar to those used as prespecified covariates (15 factors). A similar analysis was carried out 
Table 2 Baseline patient characteristics of the Japanese population and the non-Japanese population of ToGA

\begin{tabular}{|c|c|c|c|c|}
\hline \multirow[t]{2}{*}{ Characteristic } & \multicolumn{2}{|l|}{ Japanese } & \multicolumn{2}{|l|}{ Non-Japanese } \\
\hline & Trastuzumab plus XP $(n=51)$ & $\mathrm{XP} / \mathrm{FP}(n=50)$ & Trastuzumab plus XP $(n=243)$ & $\mathrm{XP} / \mathrm{FP}(n=240)$ \\
\hline \multicolumn{5}{|l|}{ Sex } \\
\hline Male, $n$ & $40(78.4 \%)$ & $40(80.0 \%)$ & $186(76.5 \%)$ & $178(74.2 \%)$ \\
\hline Median age, years (range) & $63.0(29-76)$ & $63.5(45-81)$ & $60.0(23-83)$ & $59.0(21-82)$ \\
\hline \multicolumn{5}{|l|}{ Extent of disease } \\
\hline Locally advanced, $n$ & $0(0.0 \%)$ & $1(2.0 \%)$ & $10(4.1 \%)$ & $9(3.8 \%)$ \\
\hline Metastatic, $n$ & $51(100.0 \%)$ & $49(98.0 \%)$ & $233(95.9 \%)$ & $231(96.3 \%)$ \\
\hline \multicolumn{5}{|l|}{ Primary tumor site } \\
\hline Stomach, $n$ & $49(96.1 \%)$ & $44(88.0 \%)$ & $187(77.0 \%)$ & $198(82.5 \%)$ \\
\hline Gastroesophageal junction, $n$ & $2(3.9 \%)$ & $6(12.0 \%)$ & $56(23.0 \%)$ & $42(17.5 \%)$ \\
\hline \multicolumn{5}{|l|}{ Measurability of disease } \\
\hline Measurable, $n$ & $45(88.2 \%)$ & $41(82.0 \%)$ & $224(92.2 \%)$ & $216(90.0 \%)$ \\
\hline Nonmeasurable, $n$ & $6(11.8 \%)$ & $9(18.0 \%)$ & $19(7.8 \%)$ & $24(10 \%)$ \\
\hline \multicolumn{5}{|l|}{ ECOG performance status } \\
\hline $0-1, n$ & $51(100.0 \%)$ & $50(100.0 \%)$ & $213(87.7 \%)$ & $213(88.7 \%)$ \\
\hline $2, n$ & $0(0.0 \%)$ & $0(0.0 \%)$ & $30(12.3 \%)$ & $27(11.3 \%)$ \\
\hline \multicolumn{5}{|l|}{ Chemotherapy regimen } \\
\hline $\mathrm{XP}, n$ & $51(100 \%)$ & $50(100 \%)$ & $205(84.4 \%)$ & $205(85.4 \%)$ \\
\hline FP, $n$ & $0(0.0 \%)$ & $0(0.0 \%)$ & $38(15.6 \%)$ & $35(14.6 \%)$ \\
\hline Number of lesions & & & $(n=242)$ & \\
\hline $1-4, n$ & $16(31.4 \%)$ & $18(36.0 \%)$ & $112(46.3 \%)$ & $98(40.8 \%)$ \\
\hline$>4, n$ & $35(68.6 \%)$ & $32(64.0 \%)$ & $130(53.7 \%)$ & $142(59.2 \%)$ \\
\hline Median value (range) & $6(1-15)$ & $6(1-15)$ & $5(1-20)$ & $5(1-16)$ \\
\hline Number of metastatic sites & & & $(n=242)$ & \\
\hline $1-2, n$ & $28(54.9 \%)$ & $32(64.0 \%)$ & $124(51.2 \%)$ & $114(47.5 \%)$ \\
\hline$>2, n$ & $23(45.1 \%)$ & $18(36.0 \%)$ & $118(48.8 \%)$ & $126(52.5 \%)$ \\
\hline Median value (range) & $2(1-5)$ & $2(1-5)$ & $2(1-7)$ & $3(1-8)$ \\
\hline Type of gastric cancer (central review) ${ }^{\mathrm{a}}$ & & & $(n=242)$ & $(n=237)$ \\
\hline Intestinal type, $n$ & $37(72.5 \%)$ & $42(84.0 \%)$ & $188(77.7 \%)$ & $171(72.2 \%)$ \\
\hline Diffuse type, $n$ & $5(9.8 \%)$ & $4(8.0 \%)$ & $21(8.7 \%)$ & $21(8.9 \%)$ \\
\hline Mixed type, $n$ & $9(17.6 \%)$ & $4(8.0 \%)$ & $33(13.6 \%)$ & $45(19.0 \%)$ \\
\hline \multicolumn{5}{|l|}{ Visceral metastasis (liver or lung) } \\
\hline Yes, $n$ & $35(68.6 \%)$ & $33(66.0 \%)$ & $134(55.1 \%)$ & $139(57.9 \%)$ \\
\hline No, $n$ & $16(31.4 \%)$ & $17(34.0 \%)$ & $109(44.9 \%)$ & $101(42.1 \%)$ \\
\hline \multicolumn{5}{|l|}{ History of treatment for gastric cancer } \\
\hline Prior gastrectomy, $n$ & $8(15.7 \%)$ & $13(26.0 \%)$ & $62(25.5 \%)$ & $49(20.4 \%)$ \\
\hline Prior chemotherapy, $n$ & $1(2.0 \%)$ & $0(0.0 \%)$ & $26(10.7 \%)$ & $12(5.0 \%)$ \\
\hline \multicolumn{5}{|l|}{ HER2 status } \\
\hline IHC 0/FISH-positive, $n$ & $3(5.9 \%)$ & $9(18.0 \%)$ & $20(8.2 \%)$ & $29(12.2 \%)$ \\
\hline IHC $1+/$ FISH-positive, $n$ & $10(19.6 \%)$ & $7(14.0 \%)$ & $28(11.5 \%)$ & $25(10.4 \%)$ \\
\hline IHC 2+/FISH-positive, $n$ & $18(35.3 \%)$ & $13(26.0 \%)$ & $62(25.5 \%)$ & $66(27.5 \%)$ \\
\hline IHC 3+/FISH-positive, $n$ & $16(31.4 \%)$ & $17(34.0 \%)$ & $115(47.3 \%)$ & $108(45.0 \%)$ \\
\hline IHC 3+/FISH-negative, $n$ & $1(2.0 \%)$ & $0(0.0 \%)$ & $8(3.3 \%)$ & $6(2.5 \%)$ \\
\hline IHC unknown/FISH-positive, $n$ & $0(0.0 \%)$ & $0(0.0 \%)$ & $5(2.1 \%)$ & $2(0.8 \%)$ \\
\hline IHC $3+/$ FISH unknown, $n$ & $3(5.9 \%)$ & $4(8.0 \%)$ & $5(2.1 \%)$ & $4(1.7 \%)$ \\
\hline \multicolumn{5}{|l|}{ Region of origin } \\
\hline Japanese, $n$ & $51(100 \%)$ & $50(100 \%)$ & $0(0.0 \%)$ & $0(0.0 \%)$ \\
\hline Non-Japanese, $n$ & $0(0.0 \%)$ & $0(0.0 \%)$ & $243(100 \%)$ & $240(100 \%)$ \\
\hline
\end{tabular}

ECOG Eastern Cooperative Oncology Group, FISH fluorescence in situ hybridization, HER2 human epidermal growth factor receptor 2, IHC immunohistochemistry, $X P$ capecitabine plus cisplatin

a Type of gastric cancer was described by the Lauren Classification 
using HER2 expression (high or low) as the stratification factor. The HR was approximately 0.7, and the HRs using three, four, five, and six covariates were between 0.67 (95\% CI $0.38-1.18)$ and 0.79 (95\% CI $0.46-1.39), 0.70$

Table 3 Overall survival in the Japanese population of ToGA (unadjusted Cox regression analysis)

\begin{tabular}{lll}
\hline & $\begin{array}{l}\text { Trastuzumab plus } \\
\text { XP }(n=51)\end{array}$ & $\begin{array}{l}\text { XP } \\
(n=50)\end{array}$ \\
\hline Number of events (\%) & $28(54.9)$ & $27(54)$ \\
Median OS, months (95\% CI) & $15.9(12-25)$ & $17.7(12-24)$ \\
Survival rate (\%) & & \\
6 months & 92 & 92 \\
12 months & 68 & 64 \\
18 months & 48 & 49 \\
24 months & 41 & 35 \\
Hazard ratio (95\% CI) & $1.00(0.59-1.69)$ & \\
\hline
\end{tabular}

$\overline{C I}$ confidence interval, $O S$ overall survival, $X P$ capecitabine plus cisplatin

Table 4 Preplanned multivariate Cox regression analysis of overall survival by extent of disease, primary tumor site, measurability of disease, ECOG status, chemotherapy regimen, and other prespecified

(95\% CI $0.40-1.24)$ and 0.82 (95\% CI $0.47-1.42), 0.68$ (95\% CI 0.39-1.22) and 0.76 (95\% CI 0.43-1.34), and 0.67 (95\% CI 0.37-1.22) and 0.78 (95\% CI 0.44-1.36), respectively. Influential covariates chosen in the well-fitting models included sex, prior gastrectomy, and number of lesions. Table 5 shows the covariate combinations that resulted in a good fit based on these analyses. Figure 2 shows the distribution of patient risk with these three models. The risk distribution is broad in each arm; however, the XP arm shows a somewhat greater distribution toward the left, indicating that this arm included a greater number of patients with better prognosis.

\section{Safety}

Table 6 shows the adverse events in the Japanese population of ToGA, and indicates that all patients experienced at least one adverse event in each arm. Grade 3/4 adverse events occurred in 43 patients $(84 \%)$ in the trastuzumab

covariates: sex, age, number of lesions, number of metastatic sites, type of gastric cancer, visceral metastasis, prior gastrectomy, prior chemotherapy, HER2 status, and region of origin

\begin{tabular}{|c|c|c|c|}
\hline \multirow[b]{2}{*}{ Trastuzumab plus XP versus XP } & \multicolumn{2}{|c|}{ Hazard ratio $(95 \% \mathrm{CI})$} & \multirow{2}{*}{$\frac{P \text { value }}{0.2251}$} \\
\hline & 0.68 & $(0.36-1.27)$ & \\
\hline Sex (male vs. female) & 0.16 & $(0.07-0.41)$ & $<0.0001$ \\
\hline Age $(<60$ vs. $\geq 60)$ & 1.07 & $(0.54-2.13)$ & 0.8382 \\
\hline Extent of disease (locally advanced vs. metastatic) & 0.00 & $(0.00-)$. & 0.9902 \\
\hline Primary tumor site (stomach vs. gastroesophageal junction) & 0.68 & $(0.25-1.87)$ & 0.4559 \\
\hline Measurability of disease (measurable vs. nonmeasurable) & 0.95 & $(0.29-3.05)$ & 0.9268 \\
\hline ECOG performance status & - & - & - \\
\hline Chemotherapy regimen & - & - & - \\
\hline Number of lesions (1-4 vs. $>4)$ & 0.49 & $(0.22-1.09)$ & 0.0818 \\
\hline Number of metastatic sites (1-2 vs. $>2)$ & 0.79 & $(0.41-1.50)$ & 0.4695 \\
\hline \multicolumn{4}{|l|}{ Type of gastric cancer } \\
\hline Diffuse type versus intestinal type & 3.24 & $(1.08-9.70)$ & 0.0356 \\
\hline Mixed type versus intestinal type & 0.91 & $(0.30-2.71)$ & 0.8644 \\
\hline Visceral metastasis (yes vs. no) & 1.15 & $(0.48-2.74)$ & 0.7510 \\
\hline Prior gastrectomy (yes vs. no) & 0.22 & $(0.06-0.75)$ & 0.0159 \\
\hline Prior chemotherapy (yes vs. no) & 27.72 & $(1.11-694.38)$ & 0.0432 \\
\hline \multicolumn{4}{|l|}{ HER2 status } \\
\hline IHC 0/FISH-positive versus IHC 3+/FISH-positive & 5.31 & $(1.29-21.86)$ & 0.0208 \\
\hline IHC $1+/$ FISH-positive versus IHC $3+/$ FISH-positive & 4.87 & $(1.73-13.70)$ & 0.0027 \\
\hline IHC $2+/$ FISH-positive versus IHC $3+$ /FISH-positive & 1.53 & $(0.73-3.18)$ & 0.2578 \\
\hline IHC $3+/$ FISH-negative versus IHC $3+/$ FISH-positive & 25.66 & $(1.72-382.49)$ & 0.0186 \\
\hline Region of origin & - & - & - \\
\hline
\end{tabular}

Among 15 prespecified factors, chemotherapy regimen, performance status, and region of origin were not calculated in this table because all Japanese patients received capecitabine as the chemotherapy partner of cisplatin, had Karnofsky performance status of $0-1$, and were from Asia (Japan)

CI confidence interval, ECOG Eastern Cooperative Oncology Group, FISH fluorescence in situ hybridization, HER2 human epidermal growth factor receptor 2, IHC immunohistochemistry, $X P$ capecitabine plus cisplatin 
Fig. 1 Unadjusted and adjusted hazard ratios for overall and progression-free survival. $C I$ confidence interval, $H R$ hazard ratio, $O S$ overall survival, $P F S$ progression-free survival, $X P$ capecitabine plus cisplatin
OS

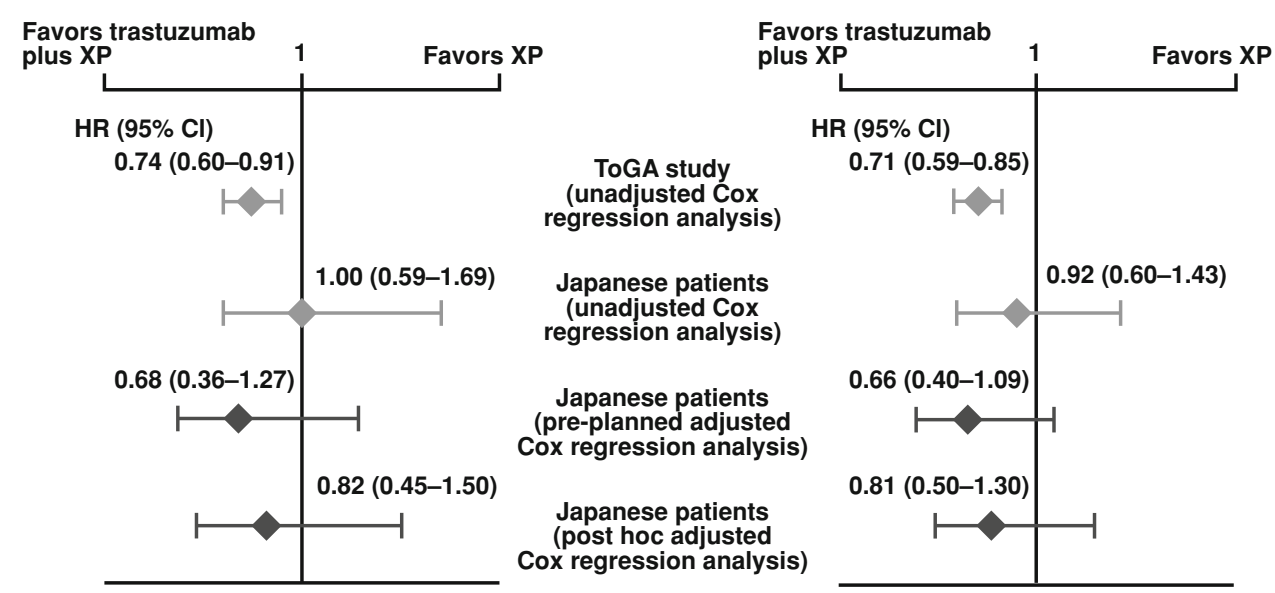

Table 5 Covariates included in the model

\begin{tabular}{ll}
\hline $\begin{array}{l}\text { Number of } \\
\text { covariates }\end{array}$ & Covariates included in the model \\
\hline 4 & HER2 expression (low/high), sex (male/female), prior gastrectomy (yes/no), number of lesions (1-4/>4) \\
5 & HER2 expression (low/high), sex (male/female), prior gastrectomy (yes/no), number of lesions (1-4/>4), type of gastric \\
cancer (diffuse/intestinal) & HER2 expression (low/high), sex (male/female), prior gastrectomy (yes/no), number of lesions (1-4/>4), type of gastric \\
6 & cancer (diffuse/intestinal), number of metastatic sites $(1-2 />2)$
\end{tabular}

HER2 human epidermal growth factor receptor 2
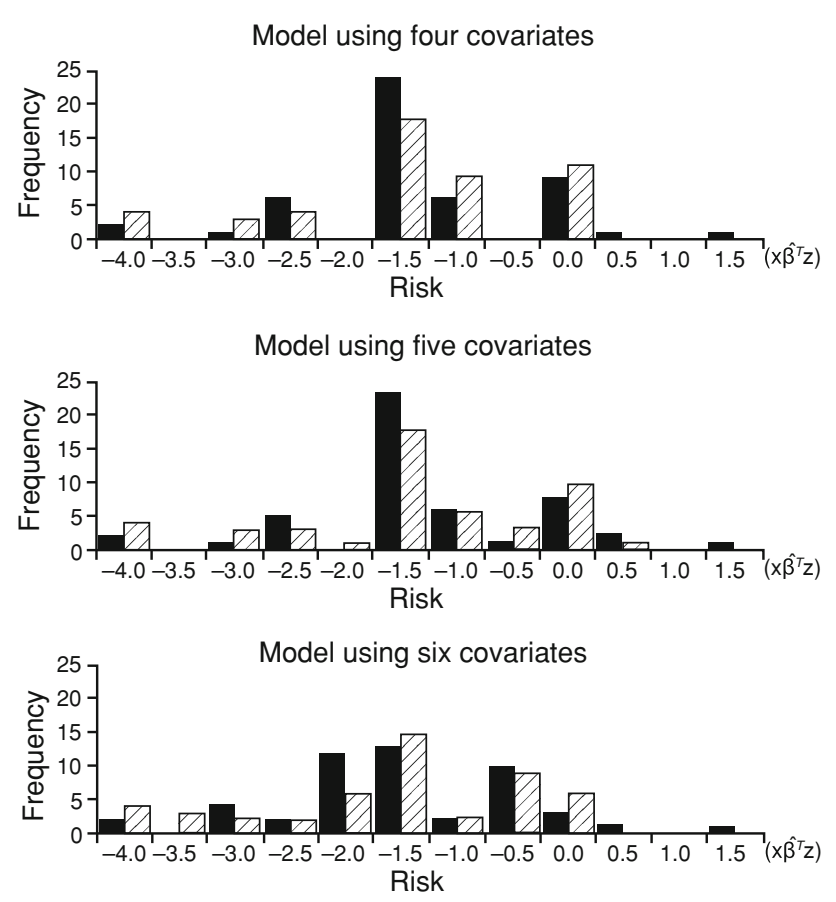

Trastuzumab plus XP $\square$ XP

Fig. 2 Distribution of estimated values by linear predictor. $X P$ capecitabine plus cisplatin. The ordinate is the number of patients and the abscissa is the risk score (estimated hazard number for each patient). The risk of mortality increases as the plot moves to the right plus XP arm and 36 patients (72\%) in the XP arm. Treatment was discontinued due to adverse events for one patient $(2 \%)$ in the trastuzumab plus XP arm and four patients $(8 \%)$ in the XP arm. Deaths due to adverse events occurred in two patients in the trastuzumab plus XP arm: one due to cardiac failure and unstable angina and the other due to gastrointestinal perforation. The case of cardiac failure and unstable angina was attributed to an adverse event likely related to trastuzumab.

\section{Discussion}

In the original ToGA study, patients with HER2-positive advanced gastric or GEJ cancer who received the combination treatment of trastuzumab plus XP/FP had significantly longer OS and PFS than patients who received XP/ FP alone [6]. No differences in OS or PFS were detected between the two treatment arms in this subgroup analysis of Japanese patients when unadjusted data were analyzed. However, in preplanned and post hoc analyses, the HRs were 0.68 and 0.82 for OS and 0.66 and 0.82 for PFS, respectively, after adjusting for baseline characteristics. These values were similar to the overall ToGA study results. Taken together, these results strongly suggest that 
Table 6 Adverse events in $\geq 10 \%$ of Japanese patients in ToGA

\begin{tabular}{|c|c|c|c|c|}
\hline & \multicolumn{2}{|c|}{ Trastuzumab plus XP $(n=51)$} & \multicolumn{2}{|l|}{$\mathrm{XP}(n=50)$} \\
\hline & All grade $n(\%)$ & Grade $\geq 3 n(\%)$ & All grade $n(\%)$ & Grade $\geq 3 n(\%)$ \\
\hline Total & $51(100)$ & $43(84)$ & $50(100)$ & $36(72)$ \\
\hline \multicolumn{5}{|l|}{ Gastrointestinal disorders } \\
\hline Nausea & $44(86)$ & $7(14)$ & $44(88)$ & $7(14)$ \\
\hline Vomiting & $33(65)$ & $1(2)$ & $28(56)$ & $2(4)$ \\
\hline Constipation & $24(47)$ & $1(2)$ & $24(48)$ & - \\
\hline Diarrhoea & $23(45)$ & $4(8)$ & $24(48)$ & $2(4)$ \\
\hline Stomatitis & $29(57)$ & - & $16(32)$ & $1(2)$ \\
\hline \multicolumn{5}{|l|}{ Blood and lymphatic system disorders } \\
\hline Neutropenia & $30(59)$ & $18(35)$ & $34(68)$ & $20(40)$ \\
\hline Thrombocytopenia & $11(22)$ & $1(2)$ & $8(16)$ & $3(6)$ \\
\hline Anemia & $15(29)$ & $13(25)$ & $11(22)$ & $8(16)$ \\
\hline Febrile neutropenia & $5(10)$ & $5(10)$ & $3(6)$ & $3(6)$ \\
\hline \multicolumn{5}{|l|}{ Skin and subcutaneous tissue disorders } \\
\hline Palmar-plantar erythrodysaesthesia syndrome & $21(41)$ & - & $23(46)$ & $1(2)$ \\
\hline Alopecia & $12(24)$ & - & $9(18)$ & - \\
\hline Skin hyperpigmentation & $6(12)$ & - & $5(10)$ & - \\
\hline Rash & $10(20)$ & - & $5(10)$ & - \\
\hline Pigmentation disorder & $10(20)$ & - & $7(14)$ & - \\
\hline Nail disorder & $5(10)$ & - & $5(10)$ & - \\
\hline \multicolumn{5}{|l|}{ Metabolism and nutrition disorders } \\
\hline Anorexia & $43(84)$ & $12(24)$ & $46(92)$ & $10(20)$ \\
\hline Dehydration & $3(6)$ & $1(2)$ & $6(12)$ & $1(2)$ \\
\hline \multicolumn{5}{|l|}{ General disorders and administration site conditions } \\
\hline Fatigue & $31(61)$ & $4(8)$ & $26(52)$ & $4(8)$ \\
\hline Pyrexia & $19(37)$ & $1(2)$ & $12(24)$ & - \\
\hline Chill & $7(14)$ & - & $0(0)$ & - \\
\hline Edema & $19(37)$ & - & $23(46)$ & - \\
\hline \multicolumn{5}{|l|}{ Nervous system disorders } \\
\hline Peripheral neuropathy & $16(31)$ & $1(2)$ & $10(20)$ & - \\
\hline Dysgeusia & $13(25)$ & - & $8(16)$ & - \\
\hline Peripheral sensory neuropathy & $2(4)$ & - & $11(22)$ & - \\
\hline Dizziness & $5(10)$ & $1(2)$ & $5(10)$ & - \\
\hline \multicolumn{5}{|l|}{ Respiratory, thoracic, and mediastinal disorders } \\
\hline Hiccups & $21(41)$ & - & $16(32)$ & - \\
\hline Epistaxis & $5(10)$ & - & $3(6)$ & - \\
\hline \multicolumn{5}{|l|}{ Renal and urinary disorders } \\
\hline Renal impairment & $32(63)$ & $2(4)$ & $27(54)$ & - \\
\hline \multicolumn{5}{|l|}{ Vascular disorders } \\
\hline Hypertension & $4(8)$ & $1(2)$ & $3(6)$ & - \\
\hline \multicolumn{5}{|l|}{ Investigations } \\
\hline Weight decreased & $27(53)$ & $2(4)$ & $13(26)$ & $1(2)$ \\
\hline Weight increased & $10(20)$ & $1(2)$ & $9(18)$ & - \\
\hline \multicolumn{5}{|l|}{ Psychiatric disorders } \\
\hline Insomnia & $11(22)$ & - & $8(16)$ & - \\
\hline \multicolumn{5}{|l|}{ Infections and infestations } \\
\hline Nasopharyngitis & $18(35)$ & - & $6(12)$ & - \\
\hline \multicolumn{5}{|l|}{ Musculoskeletal and connective tissue disorders } \\
\hline Back pain & $5(10)$ & - & $1(2)$ & - \\
\hline
\end{tabular}

$X P$ capecitabine plus cisplatin 
the same benefit of adding trastuzumab to chemotherapy was obtained in the Japanese patient subgroup as in the overall population.

In our subgroup analysis, the change in HR pre- and post-adjustment may have been due to an uneven distribution of prognostic factors between the two treatment arms. The XP arm included more patients with factors generally considered to be associated with a good prognosis (history of gastrectomy [14, 15], intestinal type cancer [16-19], and metastasis in fewer than two organs [19]). In the overall ToGA study and in the Japanese subgroup, gastric resection was shown to be the most influential factor affecting prognosis, as assessed by univariate Cox regression analyses (HRs of gastrectomy were 0.54 and 0.39 , respectively). In the Japanese subgroup, the number of patients who had undergone gastric resection in the XP arm $(n=13,26.0 \%)$ was approximately $10 \%$ higher than that of the trastuzumab plus XP $\operatorname{arm}(n=8$, $15.7 \%)$.

When multiple factors influence prognosis, different combinations of factors could affect the HR between two treatment groups. Therefore, to confirm that the HR is robust, it is necessary to analyze different combinations of factors. In this regard, we found that the HRs for OS were approximately 0.7 for all combinations of factors, thus supporting the robustness of our results.

Median OS in the XP/FP alone arm was 11.1 months (95\% CI 10-13) in the overall ToGA population [6], but was approximately 6.5 months longer in the Japanese subgroup (XP arm: 17.7 months). These findings are consistent with results of recent trials reporting longer survival for patients with gastric cancer in Japan than for patients in Europe and the USA. One possible reason for this difference is that more Japanese patients receive second-line or later treatment after the failure of first-line treatment [11-13]. In the ToGA study, more than $80 \%$ of Japanese patients in both treatment arms underwent second-line or further treatment, which was considerably higher than the overall rates of second-line treatment in the overall ToGA population ( $42 \%$ of patients in the trastuzumab plus XP/FP arm and $45 \%$ in the XP/FP arm) [6]. In the present study of Japanese patients, the OS of patients who received XP only was similar to that reported in other recent Japanese trials [2, 7, 8]. Furthermore, after adjusting for imbalances between the baseline characteristics of treatment arms, we detected an additive effect of trastuzumab among Japanese patients, similar to that of the overall population. By further exploratory analyses, we confirmed that the HRs in favor of trastuzumab were consistently observed after adjusting for prognostic factors. These findings strongly suggest that the benefits of trastuzumab were of the same magnitude in Japanese patients as in the whole study population, although the absolute length of survival was longer in the
Japanese subgroup. In conclusion, trastuzumab in combination with XP can be considered a new standard therapy for Japanese patients with HER2-positive advanced gastric or GEJ cancer.

Acknowledgments This study was sponsored by Chugai Pharmaceutical Co., Ltd. and F. Hoffmann-La Roche Ltd. We thank all of the patients and investigators who participated in the ToGA study in Japan.

Open Access This article is distributed under the terms of the Creative Commons Attribution Noncommercial License which permits any noncommercial use, distribution, and reproduction in any medium, provided the original author(s) and source are credited.

\section{References}

1. Foundation for Promotion of Cancer Research. Cancer Statistics in Japan 2010. Available from http://ganjoho.ncc.go.jp/public/ statistics/backnumber/2010_en.html. Accessed 30 June 2011.

2. Koizumi W, Narahara H, Hara T, Takagane A, Akiya T, Takagi $\mathrm{M}$, et al. S-1 plus cisplatin versus S-1 alone for first-line treatment of advanced gastric cancer (SPIRITS trial): a phase III trial. Lancet Oncol. 2008;9:215-21.

3. Hudis CA. Trastuzumab-mechanism of action and use in clinical practice. N Engl J Med. 2007;357:39-51.

4. Bang Y-J, Chung HC, Xu JM, Lordick F, Sawaki A, Lipatov O, et al. Pathological features of advanced gastric cancer: relationship to human epidermal growth factor receptor 2 positivity in the global screening programme of the ToGA trial. J Clin Oncol. 2009;27:abstract 4556.

5. Slamon DJ, Godolphin W, Jones LA, Holt JA, Wong SG, Keith DE, et al. Studies of the HER-2/neu proto-oncogene in human breast and ovarian cancer. Science. 1989;244:707-12.

6. Bang YJ, Van Cutsem E, Feyereislova A, Chung HC, Shen L, Sawaki A, et al. ToGA Trial Investigators. Trastuzumab in combination with chemotherapy versus chemotherapy alone for treatment of HER2-positive advanced gastric or gastro-oesophageal junction cancer (ToGA): a phase 3, open-label, randomized controlled trial. Lancet. 2010;376:687-97.

7. Tsuburaya $A$, Narahara $H$, Imamura $H$, Hatake $K$, Imamoto $H$, Esaki T, et al. GC0301/TOP002 Study Group. Updated result on the 2.5-year follow-up of GC0301/TOP-002: randomized phase III study of irinotecan plus S-1 (IRI-S) versus S-1 alone as firstline treatment for advanced gastric cancer (AGC). J Clin Oncol. 2009;27:abstract 4544.

8. Boku N, Yamamoto S, Fukuda H, Shirao K, Doi T, Sawaki A, et al. Gastrointestinal Oncology Study Group of the Japan Clinical Oncology Group. Fluorouracil versus combination of irinotecan plus cisplatin versus S-1 in metastatic gastric cancer: a randomized phase 3 study. Lancet Oncol. 2009;10:1063-9.

9. Van Cutsem E, Moiseyenko VM, Tjulandin S, Majlis A, Constenla M, Boni C, et al. Phase III study of docetaxel and cisplatin plus fluorouracil compared with cisplatin and fluorouracil as firstline therapy for advanced gastric cancer: a report of the V325 Study Group. J Clin Oncol. 2006;24:4991-7.

10. Cunningham D, Starling N, Rao S, Iveson T, Nicolson M, Coxon $\mathrm{F}$, et al. Capecitabine and oxaliplatin for advanced esophagogastric cancer. N Engl J Med. 2008;358:36-46.

11. Ohtsu A. Chemotherapy for metastatic gastric cancer: past, present, and future. J Gastroenterol. 2008;43:256-64.

12. Ohtsu A, Yoshida S, Saijo N. Disparities in gastric cancer chemotherapy between the East and West. J Clin Oncol. 2006;24: 2188-96. 
13. Sasako M, Inoue M, Lin JT, Khor C, Yang HK, Ohtsu A. Gastric Cancer Working Group report. Jpn J Clin Oncol. 2010;40(Suppl 1):i28-37.

14. Warneke VS, Behrens H-M, Hartmann JT, Held H, Becker T, Schwarz NT, et al. Cohort study based on the seventh edition of the TNM classification for gastric cancer: proposal of a new staging system. J Clin Oncol. 2011;29:2364-71.

15. Chau I, Norman AR, Cunningham D, Waters JS, Oates J, Ross PJ. Multivariate prognostic factor analysis in locally advanced and metastatic esophago-gastric cancer-pooled analysis from three multicenter, randomized, controlled trials using individual patient data. J Clin Oncol. 2004;22:2395-403.

16. Yoshida M, Ohtsu A, Boku N, Miyata Y, Shirao K, Shimada Y, et al. Long-term survival and prognostic factors in patients with metastatic gastric cancers treated with chemotherapy in the Japan
Clinical Oncology Group (JCOG) study. Jpn J Clin Oncol. 2004;34:654-9.

17. Adachi Y, Yoshida K, Inomata M, Sato K, Shiraishi N, Kitano S, et al. Pathology and prognosis of gastric carcinoma. Well versus poorly differentiated type. Cancer. 2000;89:1418-24.

18. Sasako M, Sano T, Yamamoto S, Kurokawa Y, Nashimoto A, Kurita A, et al. Japan Clinical Oncology Group. D2 lymphadenectomy alone or with para-aortic nodal dissection for gastric cancer. N Engl J Med. 2008;359:453-62.

19. Lee SS, Lee JL, Ryu MH, Chang HM, Kim TW, Kang HJ, et al. Combination chemotherapy with capecitabine $(\mathrm{X})$ and cisplatin (P) as first line treatment in advanced gastric cancer: experience of 223 patients with prognostic factor analysis. Jpn J Clin Oncol. 2007;37:30-7. 